\title{
Efficacy of the WATERinMOTION Aquatics Exercise Programme on the Body Weight and Composition of Sedentary Older Women with Overweight/Obesity
}

\author{
Mohammadreza Rezaeipour ${ }^{1}$, Gennady Leonidovich Apanasenko², Zahra Raghi ${ }^{3}$ \\ Affiliations: 'University of Sistan and Baluchestan, Faculty of Educational Sciences and Psychology, Department of Sports Science, Zahedan, Iran, \\ ${ }^{2} \mathrm{NMAPE}$, Faculty of sports medicine, Ukrainian Center of Sports Medicine, Kyiv, Ukraine, ${ }^{3}$ University of Sistan and Baluchestan, Faculty of Educational \\ Sciences and Psychology, Department of Sports Science, Zahedan, Iran
}

Correspondence: M. Rezaeipour, University of Sistan and Baluchestan, Faculty of Educational Sciences and Psychology, Department of Sports Science, 9816745639, Zahedan, Iran. E-mail: rezaeipour@ped.usb.ac.ir

\begin{abstract}
Some controversy remains regarding the effects of aquatic exercise on body weight and composition. The purpose of this study was to determine the short-term impacts of the WATERinMOTION aquatics exercise program on body weight and composition without nutritional intervention in sedentary older women with overweight/ obesity. The study was developed as a quasi-experimental project (pre/post-study). Forty-four inactive women were volunteers from a convenience sample with a mean age of $71.1 \pm 5.7$ years (Ukraine, 2019). Participants were allocated randomly into two groups: WATERinMOTION $(n=22)$ or a control group $(n=22)$. Meanwhile, the WATERinMOTION group performed the WATERinMOTION exercise programme with two weekly sessions of 55 minutes each. The control subjects did not participate in any physical exercises. They were asked to perform their routine activities during the study. Both programmes lasted one month. Height, body weight, body mass index (BMI), waist circumference (WC), fat mass (FM), total body water (TBW), and free fat mass (FFM) were the anthropometric variables that were measured pre/post-study. The analysis found statistically significant differences in body weight $(-0.7, \mathrm{P}=0.004), \mathrm{BMI}(-0.3, \mathrm{P}=0.002)$, and $\mathrm{FM}(-0.6, \mathrm{P}=0.03)$ between the pre and post measurements of the WATERInMOTION group. Moreover, the comparison of groups at post revealed a significant difference in body weight $(P<0.001)$, BMI $(P<0.001)$, TBW $(P=0.005)$, FM $(P<0.001)$, FFM $(P=0.003)$, and WC $(P=0.007)$. The WATERinMOTION programme, which is not associated with nutritional monitoring, showed significant benefits for losing weight and the body composition of sedentary older women who are overweight and/or obese.
\end{abstract}

Keywords: body composition, exercise therapy, resistance training, waist circumference, obesity, weight loss

$@ M J S S M o n t e n e g r o$

THE WATERINMOTION PROGRAMME AND OBESITY

http://mjssm.me/?sekcija=article\&artid $=218$

Cite this article: Rezaeipour, M., Apanasenko, G.L., \& Raghi, Z. (2021). Efficacy of the WATERinMOTION Aquatics Exercise Programme on the Body Weight and Composition of Sedentary Older Women with Overweight/Obesity. Montenegrin Journal of Sports Science and Medicine, 10 (2), 19-24. doi: 10.26773/mjssm.210903

\section{Introduction}

The global obesity epidemic is becoming worse, and reduced physical activity or increased energy consumption are two effective factors in this regard (Church \& Martin, 2018). Atherosclerosis, stroke, hyperlipidemia, certain malignancies, and sleep disturbances are some of the serious health risks 
associated with obesity (Kivimäki et al., 2017). Ageing is a natural occurrence, but the incidence of obesity in the elderly has doubled in recent years (Vuittonet et al., 2020). Along with serious risks for health, obesity can lead to chronic painful states which are comorbid during ageing (Vuittonet et al., 2020). Physical exercise, in addition to increasing the number of calories consumed by the body to gain energy (Rezaeipour, 2019; Rezaeipour \& Apanasenko, 2020), is also important for those suffering from chronic pain (Geneen et al., 2017). Currently, body mass index (BMI) is used to determine the normal weight and recognition of underweight, overweight, and obese people (World Health Organization, 2000). However, BMI measurements cannot separately quantify the composition of body fat (Nowak et al., 2019). Some people may have a BMI that places them in the obese category but have no excess body fat. Accordingly, BMI along with the measuring of body fat instead of merely measuring body weight is preferable to determine the possible health risks to an individual due to various adverse health outcomes (Nowak et al., 2019). Bioelectrical impedance analysis (BIA) is a quick, non-invasive, simple, and inexpensive method, as well as a good tool to evaluate body fat (Rezaeipour \& Apanasenko, 2019).

When a person attempts to reduce body weight or keep healthy body weight, exercise is especially important. However, some persons have restrictions that may limit their ability to take part in land-based exercise programs; for example, those who are obese or have low levels of fitness, as well as people with mobility difficulties due to senility, neuro-skeletal dysfunctions, and pulmonary disorders (Neiva et al., 2018). Raffaelli et al. (2010) found that aquatic exercise compared to land-based exercise led to noticeable rises in physical activity. Aquatic exercise is defined as a low-impact activity that occurs in water (Sanders et al., 2016), which, due to greater resistance than air, increases heart rate and energy flow and includes movements of the whole body (Tamin \& Loekito, 2018).

At the same time, literature reviews confirm the usage of aquatic exercise to promote body weight and composition (Penaforte et al., 2015; Pereira et al., 2018; Rezaeipour \& Apanasenko, 2019). Other researchers do not accept the effect of water exercises on body weight (Charmas \& Gromisz, 2019) and composition (Charmas \& Gromisz, 2019; Rica et al., 2013; Siqueira et al., 2017), and there is still controversy over this issue (Rezaeipour, 2020). Also, no literature has been found that would specifically assess the efficacy of the WATERinMOTION aquatic exercise programme on body weight and composition. The WATERinMOTION programme is a standardized aquatic exercise program with music that contains a warm-up, linear and lateral movements, team formation, dynamic group workouts, upper and lower body suspension, strengthening the core body, and flexibility (Darley, 2020). This music, in particular, is adapted so that people of any age can take part in it and the choreography fits the music. While other aquatic exercise studies have had at least a six-week (Ferrigan et al., 2017) or longer programmes (Neiva et al., 2018; Rezaeipour, 2020; Rezaeipour \& Apanasenko, 2019; Rezaeipour \& Nychyporuk, 2019), this study investigated whether two weekly sessions per month would be enough to result in significant health benefits.

The purpose of this study was to define whether the shortterm WATERinMOTION aquatics exercise programme is effective in promoting participants' body weight, waist circumference (WC), and altering body composition. It was hy- pothesized that the WATERinMOTION, which is designed to be used for approximately one month, may be a good option for promoting weight reduction, WC, and body composition of sedentary older women who are overweight and/or obese.

\section{Methods}

\section{Participants and study design}

Forty-four participants (mean age of $71.1 \pm 5.7$ years) of this quasi-experimental study were taken from a convenience sample of women volunteered to study at the Ukrainian Centre for Sports Medicine, Kyiv, in 2019. A call from a website was used to inform potential participants. With the probability of a population of 49 people, a $95 \%$ confidence level, and a five-confidence interval, a sample size of at least 44 would have been desirable, according to Creative Research Systems (2012). The research team, which included two physicians and two trainers, explained the study to the participants. A medical examination was carried out, and participants presented a physician's permit, which confirmed that the study was not prohibited for participants.

The participants were allocated randomly into two groups: WATERinMOTION $(n=22)$ or a control group $(n=22)$. The WATERinMOTION group performed the WATERinMOTION exercise programme with two weekly sessions of 55 minutes each. The control subjects did not participate in any physical exercises; they were asked to perform their routine activities during the study. The random distribution was made by a manual lottery in which individuals cast their lotteries (Rezaeipour \& Apanasenko, 2019). Both programs lasted one month. Participants voluntarily consented and participate based on the following inclusion criteria: sedentary lifestyle as physical exercise frequency from one to two times a month or less (Rezaeipour \& Nychyporuk, 2019), women aged 65 and over, BMI of $\geq 25 \mathrm{~kg} . \mathrm{m}-2$ (Rezaeipour, 2020; Rezaeipour \& Apanasenko, 2019), living self-sufficiently and classified with the level of the functional status of 3, 4 or 5 on the Basic Activities of Daily Living scale (the average status was three in this study) (Spirdus et al., 1995). The assessment of the cognitive status of more than 18 on the Mini-Mental State Examination was another inclusion criterion (Ogden et al., 2006). Exclusion criteria included weight instability ( $\pm 2 \mathrm{~kg}$ ) in the previous three months, blood pressure greater than $140 / 85 \mathrm{mmHg}$, missing three consecutive exercise sessions or more than a third of sessions, and a history of weight control supplements or medications (such as hormone replacement therapy). Blood pressure was assessed based on recommendations of the European Society of Hypertension (O'Brien et al., 2005) using a validated Omron M10-IT (HEM-7080IT-E, Kyoto, Japan) sphygmomanometer. The protocol of the study with all relevant strategies and institutional policies was drawn up under the Helsinki Declaration. The Institutional Research Ethics Committee approved this study and its informed consent forms.

\section{WATERIIMOTION program}

The typical WATERinMOTION program consisted of five minutes of warm-up and 45-minute of low-impact, high-energy cardio training, which consisted of linear and lateral suspensions, upper and lower body movements, strengthening the core body, flexibility, and five final minutes of cooling down (Darley, 2020). Supplementary Materials (Figure 1 and Table 1) are provided for a more detailed explanation of WATERinMOTION. 


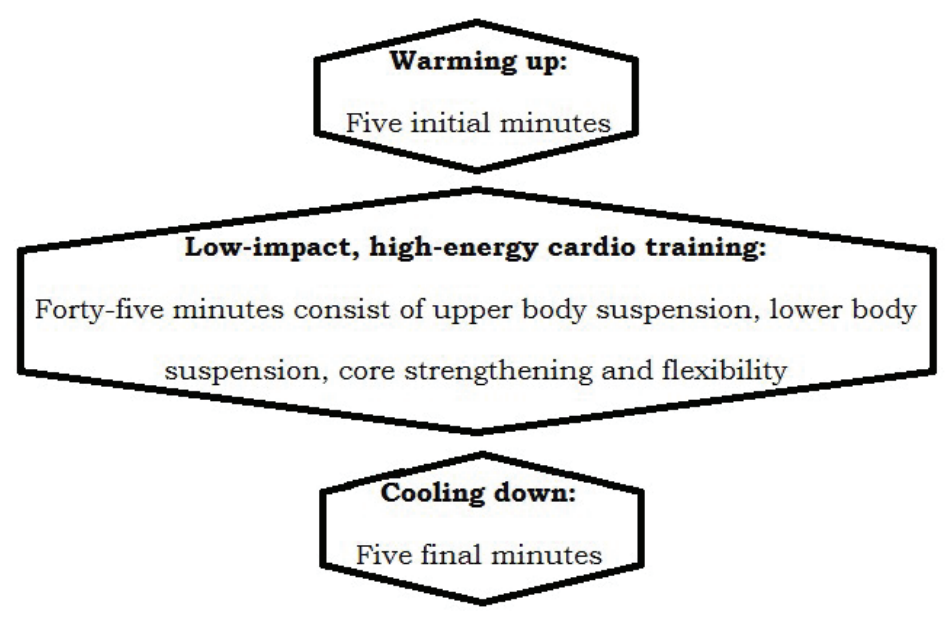

Figure 1. The organizational structure of the aquatic exercise program of WATERinMOTION

Additionally, the music used for the WATERinMOTION is specifically adapted so that people of any age can take part in it, and the choreography fit the music (Darley, 2020). The conditioning of intensity was considered moderate to vigorous or 70 $85 \%$ of the maximum heart rate (Rezaeipour, 2020). Participants' heart rates were checked with a waterproof heart rate monitor (Polar Electro Oy, Kempele, Finland) at all the training sessions. The typical water fitness temperature is $28-30{ }^{\circ} \mathrm{Celsius}$ (Bure, 2019), and the pool depth was $1 / 30 \mathrm{~m}$. The sessions of the WATERinMOTION program were carried out in an indoor pool.

Table 1. The aquatics exercise program of WATERinMOTION

\begin{tabular}{|c|c|c|}
\hline \multicolumn{3}{|r|}{ Warm-up (5 min) } \\
\hline \multicolumn{3}{|c|}{ Gradual training of the body and mind with cardio moves to enter the main phase of the exercise program } \\
\hline \multicolumn{3}{|r|}{ Main exercise ( $45 \mathrm{~min})$} \\
\hline \multirow{3}{*}{ Stage 1} & Upper-body suspension & Focusing on the upper body muscles with different levels of exercise using the next song. \\
\hline & Lower-body suspension & $\begin{array}{l}\text { Improving the range of motion, increasing the heart rate slowly, strengthening the cardiovascular } \\
\text { system and balance using aqua dumbbells, and exercising the muscles of the buttocks, thighs, hips, } \\
\text { ABS, and back through resistance training. }\end{array}$ \\
\hline & Core strengthening & Exercising the abdominal and back muscles. \\
\hline \multirow{3}{*}{ Stage 2} & Upper-body suspension & $\begin{array}{l}\text { Increasing the strength, tone, and endurance in the muscles of the chest, back, biceps, triceps, and } \\
\text { shoulders using one or both aqua dumbbells. }\end{array}$ \\
\hline & Lower-body suspension & $\begin{array}{l}\text { Continuing the resistance training and increasing the heart rate and core temperature again, using } \\
\text { the aqua dumbbells and high-intensity, low-impact movements. }\end{array}$ \\
\hline & Core strengthening & $\begin{array}{l}\text { Returning to the power centre and enhancing tone and tighten the middle section of the body with } \\
\text { exercise. }\end{array}$ \\
\hline Stage 3 & Flexibility & Enhancing the mobility and flexibility of the whole body with the last sports music track. \\
\hline \multicolumn{3}{|r|}{ Cool-down (5 min) } \\
\hline & & Easy stretching and relaxation exercises \\
\hline
\end{tabular}

\section{Body measurements}

Participants' anthropometric measures of body weight in kilogram $(\mathrm{kg})$, height in centimetres $(\mathrm{cm})$, and body composition obtained before starting the first aquatic exercise session by the research teams, and their BMI was computed from this data. Both pre/post measures of participants' weight (with a Scale-Tronix model 5002, Wheaton, IL, USA) and height (with a stadiometer) were conducted with the accuracy of $0.1 \mathrm{~kg}$, and 0.1 $\mathrm{cm}$, respectively (Rezaeipour \& Apanasenko, 2019). These measures were taken by participants dressed in light clothing and without footwear. The BMI formula is "the weight of a person in kilogram, which is divided into the height squares in meter" (CDC, 2017). A BMI of 25.0 or higher is defined as overweight, and a BMI of 30 or more is defined as obese (CDC, 2017). The WC measurement was carried out in the horizontal plane at the middle point between the crest of the ilium and the last rib at the end of a normal exhalation (Rezaeipour \& Nychyporuk, 2019).

\section{Analysis of body composition}

The composition of the body was estimated by tetrapolar BIA (BIA 310A, Biodynamics, USA), which gives data on total body water (BW), fat-free mass (FFM), and fat mass (FM) (Rezaeipour, 2020; Rezaeipour \& Apanasenko, 2019). The reliability of these data are accepted (Mainenti et al., 2011). Before the BIA test, these recommendations were emphasized for each person: do not drink alcohol or caffeine for 48-hour before the test; do not participate in physical exercise within 24-hour before the test; do not consume food or beverages before the test; do not take diuretics for seven days before the beginning of the evaluation (Pereira et al., 2018). The FFM prediction was conducted using data provided by the BIA based on the equation of Gray et al. (Gray et al., 2018) for the elderly, which is 
as follows:

FFM $($ in $\mathrm{Kg})=0.00151$ (height2 in $\mathrm{cm})-0.0344$ (resistance in ohms) +0.14 (body weight in $\mathrm{Kg}$ ) -0.158 (age in full years) +20.387 ; FM (in kg) = body weight - FFM.

To control eating habits and drink intake, a diary was used, which had previously been approved for use in households (Rezaeipour, 2020; Rezaeipour \& Apanasenko, 2019). Participants were instructed to complete their dietary records within 24-hours of four days (three days a week and weekend day), and the same method was carried out immediately following the study to report altered eating habits and drink intake during the study period (Rezaeipour, 2020; Rezaeipour \& Apanasenko, 2019). All participants were asked to maintain their dietary habits, drink intake, and leisure-time physical activities throughout the study. Each of the participants was tracked every two-week.

\section{Statistical analysis}

Data entry and statistical analysis were utilizing IBM SPSS 21.0 (SPSS Inc. USA) for Windows. Normally distributed of data was verified using the Shapiro-Wilk test and expressed in the form of mean values $\pm \mathrm{SD}$. Comparing the pre- and poststudy was carried out using research data analysis with paired samples t-test and a Bonferroni correction. Comparisons between groups were carried out using research data analysis by Analysis of Covariance (ANCOVA). The results were corrected using the Bonferroni correction. The mean differences were statistically significant if the P-values were less than 0.05 . Bonferroni correction was computed by dividing alpha by the number of analyses performed. The statistically significant levels decreased from 0.05 to 0.008 . Linear regression analysis was applied to study a relation between all significant (dependent) variables and changes in body weight (independent).

\section{Results}

No side effects and dropouts were reported during the study. Forty-four participants who met the study criteria completed the study. Table 2 shows the variables evaluated in the study groups pre- and post-study.

Table 2. Mean of the Studied Groups' Variables Pre- and Post-Study

\begin{tabular}{ccccc}
\hline \multirow{2}{*}{ Variables } & \multicolumn{2}{c}{ WATERinMOTION Group } & \multicolumn{2}{c}{ Control Group } \\
\cline { 2 - 4 } & Group Mean Pre-Study & Group Mean Post-Study & Group Mean Pre-Study & Group Mean Post-Study \\
\hline Body weight $(\mathrm{kg})$ & $85.5 \pm 13.8$ & $84.8 \pm 14.3^{*}$ & $85.9 \pm 15.3$ & $86.1 \pm 15.1$ \\
BMI (kg.m $\left.{ }^{-2}\right)$ & $33.4 \pm 5.5$ & $33.1 \pm 5.8^{*}$ & $33.5 \pm 5.8$ & $33.6 \pm 5.7$ \\
TBW $(\mathrm{L})$ & $35.5 \pm 6.4$ & $35.3 \pm 6.4$ & $35.6 \pm 6.9$ & $35.7 \pm 6.8$ \\
FM $(\mathrm{kg})$ & $35 \pm 7.8$ & $34.4 \pm 8.1^{*}$ & $35.2 \pm 8.5$ & $35.3 \pm 8.4$ \\
FFM (kg) & $48.5 \pm 8.9$ & $48.5 \pm 9.1$ & $48.7 \pm 9.6$ & 0.005 \\
WC $(\mathrm{cm})$ & $87.2 \pm 9.3$ & $87 \pm 8.79$ & $87.6 \pm 10.1$ & $48.9 \pm 9.4$ \\
\hline
\end{tabular}

Note. Data are reported as mean \pm SD; BMI: body mass index; TBW: total body water; FM: fat mass; FFM: fat-free mass; WC: waist circumference; *: P-value < 0.008 in comparison with pre-study; \#: Differences between groups.

As can be seen from Table 2, differences between the groups of the studied variables were not significant at prestudy $(\mathrm{P}>0.05)$. From the point of view of time (pre- and poststudy), significant interaction influences were found in body weight $(-0.7, \mathrm{P}=0.004)$, BMI $(-0.3, \mathrm{P}=0.002)$, and FM (-0.6, $\mathrm{P}=0.003)$ of the WATERinMOTION group. Moreover, the comparison of groups at post revealed a significant difference in all the variables studied (Table 2).

The regression analysis disclosed a significant relationship between BMI, FM, and changes in body weight. The regression coefficient in predicting the body weight variable was 0.342 $(\mathrm{P}=0.001)$ and $0.685(\mathrm{P}<0.0001)$ for $\mathrm{BMI}$ and $\mathrm{FM}$, respectively.

\section{Discussion}

The present study examined the short-term effects of the WATERinMOTION aquatic exercise program without nutritional monitoring on body weight and composition in sedentary overweight and/or obese older women; it has shown that there is a link between the WATERinMOTION programme and significant alterations in body weight and composition.

Kirsten et al. (2017) carried out a six-week program of the water aerobics and concluded that aerobic exercise in water could reduce body weight and FM in the middle-aged population. Penaforte et al. (2015) carried out an aquatic exercise programme that lasted two months with three weekly sessions in obese older women and observed a significant decline in body weight, BMI, and FM. Pereira et al. (2018) conducted an aquatic exercise program (12 weeks) in older adults and recorded reduced body weight and loss of FM. Rezaeipour and Nychyporuk (2019) conducted a 12-week water aerobics programme (three-session a week) in postmenopausal females and reported a significant reduction in weight loss parameters. Furthermore, other studies by Rezaeipour (2020) and Rezaeipour and Apanasenko (2019) on pool workouts for three months revealed a significant decline in body weight, BMI, and FM.

In contrast, Rica et al. (2013) conducted three sessions per week of a water aerobics programme for 12 weeks in obese older women. They did not see any changes in body composition parameters, but improvements in all function parameters such as arm flexion, walking time, and quality of life were reported. Siqueira et al. (2017) found no changes in the body composition of women with rheumatoid arthritis who participated in 16 weeks supervised programme comprising various types of water exercise in the form of three weekly sessions. Charmas et al. (2019) carried out swimming training, which lasted twelve weeks with three-time per week, in young women; mo changes were found in body weight and FM.

As can be observed, disagreement about the influence of aquatic exercises on body weight and composition remains. The novel finding of our study is that body weight, BMI, and FM were decreased while surprisingly the duration of the WATERinMOTION program is about four weeks compared to the standard six, twelve, or more. The significant associations found in this study with reduced body weight, BMI, and FM support the study hypothesis and suggest that using a WATER- 
inMOTION programme can yield some of the desired results on body weight and composition parameters for clinical use and show that it can be used clinically to achieve the desired results within a shorter time frame. Also, the WATERinMOTION program can be used as an alternative form of physical conditioning because of the benefits of water buoyancy and a reducer of obesity impacts.

Any type of exercise that leads to calorie-burning increases the likelihood of alterations in body composition. Water aerobics does this with internal resistance to water viscosity and creates pressure in any movement (Tamin \& Loekito, 2018). Thus, the moderate weight loss experienced during the WATERinMOTION aquatic exercise was most likely induced by a lack of compensatory increase in calorie intake to match energy expenditure during training. In fact, from the findings of this study, it can be inferred that maintaining a calorie deficit with an aquatic exercise programme will promote a positive impact on the composition of the body.

Moreover, blood flow and bone density during muscle activity are affected by FMM and are directly related to the ability to exercise (Lee \& Oh, 2015). This study showed that there was no statistically significant difference in FMM and the absence of a significant reduction in this parameter may be due to the shorter duration of the WATERinMOTION program than other studies.

Obese people, even when exercising in water, have problems with mobility and overcoming water resistance (Penaforte et al., 2015; Rezaeipour, 2020; Rezaeipour \& Apanasenko, 2019). Thus, to obtain a more significant effect on body weight and on other variables under study, the intensity and speed of activity may not be enough, because both are directly connected to exercise outcomes. Increased WC due to visceral fat may be a risk factor that causes many disorders, such as arthritis and cardiometabolic (Carr et al., 2004). The result of WC was positive but insignificant for the WATERinMOTION group. Another study conducted on older people showed a greater reduction of the WC when water-walking and also of longer duration (24 to 48 weeks) (Naylor et al., 2020), which can explain its better result than the current one.

Although the results of the project seem promising, caution should be advised concerning various limitations. The study used a convenience sample of older women, and generalizations are thus limited, and these results may not be seen outside older women due to gender and age-related metabolic differences. The four-week time frame of the WATERinMOTION aquatics exercise programme and its isolated use without nutritional monitoring to assess changes in body weight and composition may have been other limiting factors for the programme's effectiveness. Also, this study was not able to provide a comparability analysis of ground exercises or other water exercise programs.

More research in the future may examine the WATERinMOTION aquatics exercise program to support its influences on body composition and other additional health benefits. Suggestions for future studies include the use of randomized controlled trial assignment and sampling in such a way that the population under study had the chance to be selected. It is also advisable to use a larger sample and a greater duration of exercise (more than one month). The study of the WATERinMOTION aquatics exercise without nutritional monitoring is also recommended in other sexes and ages with a sedentary lifestyle. Moreover, studying the diet-restricted WATERin-
MOTION is encouraged for overweight/obese sedentary older women and/or other genders and ages. Additional health data collection, such as resting heart rate and cardiometabolic risk factors before and after studying an aquatic exercise programme, may also be useful to examine.

In conclusion, despite the sessions being held only twice a week for one month, the WATERinMOTION aquatics program, which included no nutritional monitoring, showed significant benefits for losing weight and body composition of sedentary older women who are overweight and/or obese. The results of this study will enable health professionals, such as physicians and trainers, to recommend the WATERinMOTION to their patients who cannot easily exercise on the ground to help them achieve to their physical fitness and health goals.

\section{Acknowledgement}

Thanks to my wife and the staff of the Sports Medicine Center for their kind support.

\section{References}

Bagherian, S., Ghasempoor, K., Rahnama, N., \& Wikstrom, E. A. (2019). The Effect of Core Stability Training on Functional Movement Patterns in College Athletes. Journal of Sport Rehabilitation, 28(5), 444-449. doi: 10.1123/ jsr.2017-0107

Bure, A. (2019). Physical Activity in the Water and the Human Body. Retrieved from https://www.theseus.fi/ handle/10024/167487

Carr, D. B., Utzschneider, K. M., Hull, R. L., Kodama, K., Retzlaff, B. M., Brunzell, J. D., et al. (2004). Intra-abdominal fat is a major determinant of the National Cholesterol Education Program Adult Treatment Panel III criteria for the metabolic syndrome. Diabetes, 53(8), 2087-2094. doi: 10.2337/diabetes.53.8.2087

Centers for Disease Control (2017). About Adult BMI. Retrieved from https://www.cdc.gov/healthyweight/ assessing/bmi/adult_bmi/index.html

Charmas, M., \& Gromisz, W. (2019). Effect of 12-week swimming training on body composition in young women. International journal of environmental research and public health, 16(3), 346. doi: 10.3390/ijerph16030346

Church, T., \& Martin, C. K. (2018). The obesity epidemic: a consequence of reduced energy expenditure and the uncoupling of energy intake? Obesity, 26(1), 14-16. doi: 10.1002/oby.22072

Darley, T. (2020). Effectiveness of an Aquatic Exercise Program for Reducing Weight, Body Fat and Chronic Low Back and Joint Pain. Unpublished Scholarly Project. Marquette, MI: Northern Michigan University.

Geneen, L. J., Moore, R. A., Clarke, C., Martin, D., Colvin, L. A., \& Smith, B. H. (2017). Physical activity and exercise for chronic pain in adults: an overview of Cochrane Reviews. Cochrane Database of Systematic Reviews, 1(1), CD011279. doi: 10.1002/14651858.CD011279.pub2.

Gray, D. S., Bray, G. A., Gemayel, N., \& Kaplan, K. (1989). Effect of obesity on bioelectrical impedance. The American journal of clinical nutrition, 50(2), 255-260. doi: 10.1093/ ajcn/50.2.255

Kirsten Ferrigan, K., Hice, J., Leemkuil, K., Singer, S., Charles, D., \& Michaels, N. ve Jones, T. (2017). Aquatic exercise for weight reduction in middle-aged adults: a pilot study. The 
Journal of Aquatic Physical Therapy, 25(2), 16-21.

Kivimäki, M., Kuosma, E., Ferrie, J. E., Luukkonen, R., Nyberg, S. T., Alfredsson, L., et al. (2017). Overweight, obesity, and risk of cardiometabolic multimorbidity: pooled analysis of individual-level data for 120813 adults from 16 cohort studies from the USA and Europe. The Lancet Public Health, 2(6), e277-e285. doi: 10.1016/S2468-2667(17)30074-9

Lee, B.-A., \& Oh, D.-J. (2015). Effect of regular swimming exercise on the physical composition, strength, and blood lipid of middle-aged women. Journal of exercise rehabilitation, 11(5), 266. doi: 10.12965/jer.150242

Mainenti, M. R. M., Rodrigues, É. d. C., Oliveira, J. F. d., Ferreira, A. d. S., Dias, C. M., \& Silva, A. L. d. S. (2011). Adiposity and postural balance control: correlations between bioelectrical impedance and stabilometric signals in elderly Brazilian women. Clinics, 66(9), 1513-1518. doi: 10.1590/S1807-59322011000900001

Naylor, L. H., Maslen, B. A., Cox, K. L., Spence, A. L., Robey, E., Haynes, A., et al. (2020). Land-versus water-walking interventions in older adults: Effects on body composition. Journal of science and medicine in sport, 23(2), 164-170. doi: 10.1016/j.jsams.2019.08.019

Neiva, H. P., Fail, L. B., Izquierdo, M., Marques, M. C., \& Marinho, D. A. (2018). The effect of 12 weeks of wateraerobics on health status and physical fitness: An ecological approach. PloS one, 13(5), e0198319. doi: 10.1371/journal. pone.0198319. eCollection 2018

Nowak, J., Hudzik, B., Kulik-Kupka, K., Zieleń-Zynek, I., Kowalska, J., \& Zubelewicz-Szkodzińska, B. (2019). Discordance between body-mass index and body adiposity index in classification of adiposity status in the elderly. Paper presented at the 21st European Congress of Endocrinology.

O'Brien, E., Asmar, R., Beilin, L., Imai, Y., Mancia, G., Mengden, T., et al. (2005). Practice guidelines of the European Society of Hypertension for clinic, ambulatory and self blood pressure measurement. Journal of hypertension, 23(4), 697701. doi: 10.1097/01.hjh.0000163132.84890.c4.

Ogden, C. L., Carroll, M. D., Curtin, L. R., McDowell, M. A., Tabak, C. J., \& Flegal, K. M. (2006). Prevalence of overweight and obesity in the United States, 1999-2004. Jama, 295(13), 1549-1555. doi: 10.1001/jama.295.13.1549

Penaforte, F. R. O., Calhau, R., Mota, G. R., \& Chiarello, P. G. (2015). Impact of short-term water exercise programs on weight, body composition, metabolic profile and quality of life of obese women. Journal of Human Sport and Exercise, 10(4), 915-926.

Pereira, C., da Silva, R. A., de Oliveira, M. R., Souza, R. D., Borges, R. J., \& Vieira, E. R. (2018). Effect of body mass index and fat mass on balance force platform measurements during a one-legged stance in older adults. Aging clinical and experimental research, 30(5), 441-447. doi: 10.1007/ s40520-017-0796-6

Raffaelli, C., Lanza, M., Zanolla, L., \& Zamparo, P. (2010). Exercise intensity of head-out water-based activities (water fitness). European Journal of Applied Physiology, 109(5), 829-838. doi: 10.1007/s00421-010-1419-5
Rezaeipour, M. (2019). Comparison of effect of weight loss and physical exercise on the cardiac risk profiles of middleaged women with overweight: a quasi-experimental study. Urmia Medical Journal, 30(7), 548-555.

Rezaeipour, M. (2020). Investigation of Pool Workouts on Weight, Body Composition, Resting Energy Expenditure, and Quality of Life among Sedentary Obese Older Women. Montenegrin Journal of Sports Science and Medicine, 9(1), 67-72. doi: 10.26773/mjssm.200309

Rezaeipour, M., \& Apanasenko, G. (2020). Influences of Weight Loss and Physical Exercise on Lipid Panel in Overweight Middle-Aged Men. Hormozgan Medical Journal, 24(3), e100748. doi: 10.5812/hmj.100748.

Rezaeipour, M., \& Apanasenko, G. L. (2019). Effects of Waterobics Programs on Body Mass, Body Composition, and Coronary Risk Profile of Sedentary Obese Middleaged Women. Women's Health Bulletin, 6(4), 13-17.

Rezaeipour, M., \& Nychyporuk, V. I. (2019). Study of Weight Loss Parameters Among Sedentary, Overweight Postmenopausal Females Using Different Time Models of Aquafit. Hormozgan Medical Journal, 23(4).

Rica, R. L., Carneiro, R. M. M., Serra, A. J., Rodriguez, D., Pontes Junior, F. L., \& Bocalini, D. S. (2013). Effects of water-based exercise in obese older women: Impact of short-term follow-up study on anthropometric, functional fitness and quality of life parameters. Geriatrics \& gerontology international, 13(1), 209-214. doi: 10.1111/j.1447-0594.2012.00889.x.

Sanders, M. E., Islam, M. M., Naruse, A., Takeshima, N., \& Rogers, M. E. (2016). Aquatic exercise for better living on land: Impact of shallow-water exercise on older Japanese women for performance of activities of daily living (ADL). International Journal of Aquatic Research and Education, 10(1), 1. doi: 10.25035/ijare.10.01.01

Siqueira, U. S., Valente, L. G. O., de Mello, M. T., Szejnfeld, V. L., \& Pinheiro, M. M. (2017). Effectiveness of Aquatic Exercises in Women with Rheumatoid Arthritis: A Randomized, Controlled, 16-Week Intervention-the HydRA Trial. American journal of physical medicine \& rehabilitation, 96(3), 167-175. doi: 10.1097/PHM.0000000000000564

Spirduso, W., Francis, K., \& MacRae, P. (1995). Physical dimensions of aging. Human kinetics. Champaign Illinois USA.

Systems, C. R. (2012). Sample size calculator. Retrieved from https://www.surveysystem.com/sscalc.htm

Tamin, T. Z., \& Loekito, N. (2018). Aquatic versus land-based exercise for cardiorespiratory endurance and quality of life in obese patients with knee osteoarthritis: a randomized controlled trial. Medical Journal of Indonesia, 27(4), 284292. doi: $10.13181 / \mathrm{mji} . v 27 \mathrm{i} 4.2107$

Vuittonet, C. L., Sbharwal, A., \& Pitchumoni, C. (2020). Obesity in Older Adults: Pathophysiology and Clinical Implications. Geriatric Gastroenterology, 1-19. doi: 10.1007/978-3-319-90761-1_98-1

World Health Organization. (2000). Obesity: preventing and managing the global epidemic: World Health Organization. 